\title{
Prevotella Denticola Spondylitis
}

\author{
Marie Hocquart, Jean Christophe Lagier and Philippe Brouqui* \\ Infectious Diseases Hospital North APHM , IHU Mediterranean infection, Chemin des bourelys 13015 Marseille, France
}

\begin{abstract}
We report a case of a 75 year-old man who was diagnosed with a L3-L4 spondylitis with psoas abscess documented at Prevotella denticola. However, the dental origin of the bacteria, the presence of the spondylitis, and the identification on an aneurysm of the inter-atrial septum with a patent oval foramen and finally the metabolic activity of the aortic valve and teeth revealed by $18 \mathrm{~F}-$ FDG PET/ CT makes the diagnostic of endocarditis questionable. The input of the 18F- FDG PET/ CT on the diagnosis of native valve infectious endocarditis would be valuable in these clinical situations but needed more studies.
\end{abstract}

Keywords: Spondylitis; Inter-atrial septum; Oval foramen; $18 \mathrm{~F}-$ FDG PET/ CT

\section{Background}

Prevotella species are obligate anaerobic, Gram-negative, pleomorphic rods that belong from the genus Bacteroides and were renamed recently [1]. Most species of the Prevotella genus are part of the oral cavity, and they are most frequently associated with periodontitis and dental diseases especially in children. However, in rare cases, they were also isolated from other site and have been reported as causing endocarditis [2,3],cerebral abscesses [4] and soft tissues infection $[5,6]$.We report here a case of Prevotella Denticola in the context of spondilodiscitis with epiduritis, meningitis and psoas abcesse.

\section{Case Presentation}

A 75 year-old man originating from Tunisia whose main medical history was a type II diabetes, hypertension and dyslipidemia presented with a lower back pain, weight loss and tiredness lasting for one month. In Tunisia, a CT scan and MRI were performed, showing a L3 L4 spondilodiscitis associated with an epidural and a psoas abscess. Empiric treatment by Oxacillin and Gentamicin (unknown doses) was prescribed and the patient was transferred in our department. At clinical examination, the patient was slightly confused, without neurological focal deficit or meningeal syndrome. He complained of severe lumbar back pains, associated with a psoitis without sphincterial disorders. A 4/6 mitral valve murmur was discovered. The laboratory tests showed an inflammatory disorder with CRP at $267 \mathrm{mg} / \mathrm{l}$, a WBC count at $20 \mathrm{G} / \mathrm{L}, \mathrm{Hb}$ at $10.5 \mathrm{~g} / \mathrm{dl} ; 14$ blood cultures and urine culture were sterile, CSF showed 960 elements / $\mathrm{mm} 3$ with $86 \%$ of neutrophils , $6 \%$ of lymphocyts and 1500 erythrocytes/mm3, hyperproteinorachy at $5,49 \mathrm{~g} / \mathrm{l}$ and normoglycorachy. The culture of the CSF and PCR for HSV, $\mathrm{VZV}$, meningococcus, pneumococcus, enteroviruses, mycobacteria including M.tuberculosis and M avium, and Staphylococcus aureus specific gene, as well as a 16S RNA PCR were negative. Trans thoracic and Tran's esophageal echocardiography showed the presence of an aneurysm of the inter-atrial septum with a patent oval foramen. MRI confirmed a L3/L4 spondylodiscitis with a contiguous partitioned psoas abscess measuring 17 by $5 \mathrm{~cm}$. (Figure 1). We performed radio guided drainage of the abscess with microbiological analysis which grew a gram negative bacterium identified as Prevotella denticola in MALDITOF MS and 16S rRNA PCR [7,8]. A dental panoramic radiograph found multiple granulomas on teeth $\mathrm{N}^{\circ} 21,24,31,43$. Finally a 18 F-FDG PTE/CT was performed and showed multiple hyper metabolic activity foci at L3L4 level of the spine, of the left psoas muscle, jaw but especially reveal an hypermetabolic activity on the native aortic valve (Figure 2). The diagnosis of L3L4 spondylitis due to Prevotella denticola complicated with epiduritis and psoas abscess was retained.The patient
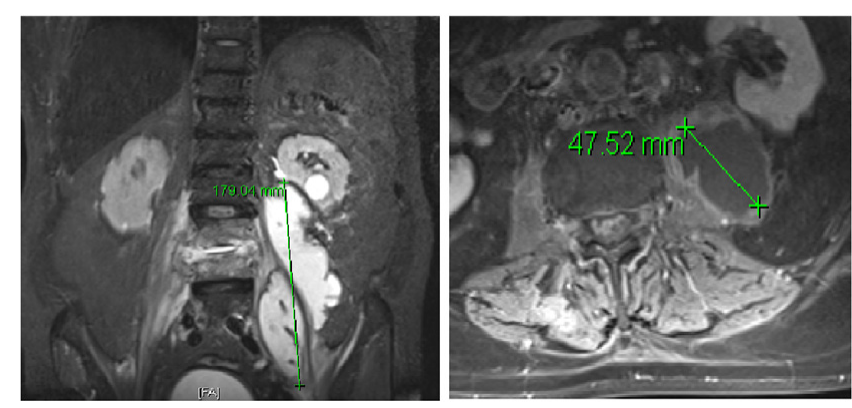

Figure 1: MRI: L3-L4 spondylitis with edema of the vertebral bodies and disc, enhanced after injection. Very large left psoas abscess interesting lumbar and iliopsoas, measuring $18 \times 5 \mathrm{~cm}$.There are also epidural abscess facing the vertebral involvement and a prevertebral spindle.
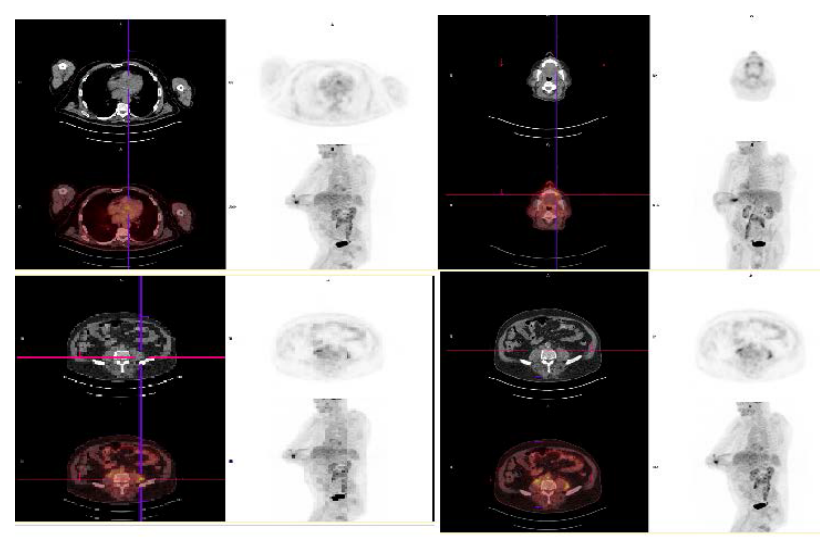

Figure 2: 18F- FDG PET/ CT : Hypermetabolism at psoas muscle in bilatera way and extended on the left from L2 to iliopsoas muscle and on the right from $\mathrm{L} 2$ to $\mathrm{L} 5$ (SUV $\max 8 \mathrm{~g} / \mathrm{ml}$ ), the L3-L4 vertebrae (SUV $\max 7 \mathrm{~g} / \mathrm{ml}$ ), maxillary and finally at the aortic valve.

*Corresponding author: Philippe Brouqui, Infectious Diseases Hospital North APHM , IHU Mediterranean infection, Chemin des bourelys 13015 Marseille, France, Tel: 029834 71 91; E-mail: philippe.brouqui@univ-amu.fr

Received August 24, 2015; Accepted September 03, 2015; Published September 10, 2015

Citation: Hocquart M, Lagier JC, Brouqui P (2015) Prevotella Denticola Spondylitis J Clin Case Rep 5: 588. doi:10.4172/2165-7920.1000588

Copyright: ( $) 2015$ Hocquart M, et al. This is an open-access article distributed under the terms of the Creative Commons Attribution License, which permits unrestricted use, distribution, and reproduction in any medium, provided the original author and source are credited. 
was treated with vancomycin (300 mg every $12 \mathrm{~h})$ and meropenem $(2 \mathrm{~g}$ every $12 \mathrm{~h}$ ) then vancomycin and imipenem ( 500 every $8 \mathrm{~h}$ ) and finally adapted to the drug susceptibility testing to amoxicillin-clavulanic acid ( $1 \mathrm{~g}$ every $8 \mathrm{~h}$ ) and metroninazol ( 500 every $8 \mathrm{~h}$ ) for a period of 3 months. The patient was followed up and both physical examination and a controlled MRI at the end of treatment shows a good evolution and the patient was considered as cured on Month 3 .

\section{Discussion}

We searched PubMed for English articles using the terms "spondylitis bacteroides OR spondylitis prevotella" because the genus Prevotella belonged to the genus Bacteroides before 1990. A total of 30 articles were identified, among which 10 were relevant to our case report; 7 cases of spondilitis due to Bacteroides fragilis, one to Prevotella melanogenica one to P.intermedia and 2 with Prevotella spp unidentified. When the terms used were "endocarditis bacteroides OR endocarditis prevotella" 135 articles were recovered among them 10 cases due to $B$. fragilis, 1 B. oralis and 4 due to Prevotella spp among one due to P. denticola [9-23]. Prevotella denticola has rarely been report in systemic infection in human. Only 2 cases of such infections are listed in the literature. A tricuspid valve endocarditis has been reported in a 34 year-old drug abuser patient HIV positive with CD4 at 74 cells / $\mathrm{mm} 3$. He did not mention recent dental manipulation history. The patient has well progressed following treatment with AmoxicillinClavulatic acid for a total of seven weeks. The authors hypothesize transmission by cleaning needles and injection sites with saliva [3]. More recently, multiple brain abscesses due to Prevotella denticola found both in accesses and in blood cultures has been reported in a young man without comorbidities [4]. The dental origin was suggested in the context of tooth extraction nine days before. He was treated by cefixime ( $2 \mathrm{~g}$ every $8 \mathrm{~h}$ ) and metronidazole( 500 every $8 \mathrm{~h}$ ), adapted to penicillin and metronidazole as recommended in brain abscesses of dental origin and relay orally with chloramphenicol ( 500 every $6 \mathrm{~h}$ ) for a total of 10 weeks. Good clinical and radiological progression thereafter. In our patient the appropriateness of the diagnosis of spondilitis can be discuss. The modified Duke criteria do not allow the diagnosis of endocarditis with only two minor criteria [24]. The patient was on antibiotics on arrival which may explain the absence of fever and bacteremia. The presence of an aneurysm of the interatrial septum with a patent oval foramen is not considered as a major diagnostic criterion but the fact that the 18 F-FDG PTE/CT reveal an hypermetabolic activity on the native aortic valve and that Prevotella spp infective endocarditis has already been reported, lead to questioned the diagnosis of infectious endocarditis. Preliminary results have been promising for 18F-fluorodeoxyglucose mock (FDG) Positron Emission Tomography (PET) / CT scans in the setting of pacemaker / defibrillator leads and prosthetic valve endocarditis [25]. On prosthetic valve, a study from our group demonstrated that 18F- FDG PET/ CT has a good diagnostic value, especially when abnormal FDG uptake around the prosthetic valve was added as a major criterion to the modified Duke classification established within a few days after admission. The inclusion of this new criterion significantly increased the sensitivity (70 to $97 \%$ ) of the modified duke classification and allowed for an earlier diagnosis, especially when echocardiography was normal or doubtful [26]. The interest of 18F- FDG PET/ CT has not yet been fully assessed in the diagnosis of native valve endocarditis except in the context of C.burnetti endocarditis [27]. To conclude, the already described involvement of Prevotella spp in endocarditis, the dental origin of the bacteria, the presence of a spondylosdicitis, the identification on an aneurysm of the inter-atrial septum with a patent oval foramen and finally the metabolic activity of the aortic valve and teeth revealed by
18F- FDG PET/ CT must evoke the diagnosis of endocarditis with spinal secondary location. The input of the 18F- FDG PET/ CT on the diagnosis of native valve infectious endocarditis would be valuable in these clinical situations but needed more studies.

\section{References}

1. Shah HN, Collins DM (1990) Prevotella, a new genus to include Bacteroides melaninogenicus and related species formerly classified in the genus Bacteroides. Int J Syst Bacteriol 40: 205-258.

2. Quaglio G, Anguera I, Miro JM (1999) Prevotella oralis homograft-valve endocarditis complicated by aortic-root abscess, intracardiac fistula, and complete heart block. Clin Infect Dis 28: 685-686.

3. Dominguez-Castellano A, Angel MM, Jose Rios-Villegas M (2001) Prevotella denticola endocarditis in an intravenous drug abuser. Enferm Infecc Microbiol Clin 19: 280-281.

4. Wu PC, Tu MS, Lin PH, Chen YS, Tsai HC (2014) Prevotella brain abscesses and stroke following dental extraction in a young patient: a case report and review of the literature. Intern Med; 53: 1881-1887.

5. Bancescu G, Dumitriu S, Bancescu A, Preoteasa E, Skaug N (2004) Prevotella species involved in the onset of superficial face and neck abscesses. Bacteriol Virusol Parazitol Epidemiol 49: 131-136.

6. Matto J, Asikainen S, Vaisanen ML (1997) Role of Porphyromonas gingivalis, Prevotella intermedia, and Prevotella nigrescens in extraoral and some odontogenic infections. Clin Infect Dis 2: 194-198.

7. Seng P, Drancourt M, Gouriet F (2009) Ongoing revolution in bacteriology: routine identification of bacteria by matrix-assisted laser desorption ionization time-of-flight mass spectrometry. Clin Infect Dis Aug 15; 49: 543-551.

8. Berger P, Adekambi T, Mallet MN, Drancourt M (2005) Prevotella massiliensis sp. nov. isolated from human blood. Res Microbiol 156: 967-973.

9. Han IH, Choi BK, Nam KH, Kim SY(2013) Bacteroides fragilis vertebral osteomyelitis complicated by percutaneous epidural adhesiolysis. Spine 38 431-435.

10. Kawakami T, Suzuki H, Suzuki M, Hirose $Y$ (2012) Spondylodiscitis complicated by an epidural abscess and meningitis caused by Bacteroides fragilis. Intern Med 51:189-191.

11. 11. Purushothaman B, Lakshmanan P, Gatehouse S, Fender D (2010) Spondylodiscitis due to Prevotella associated with ovarian mass-a rare case report and review of literature. World Neurosurg 73: 119-122.

12. Tsuji Y, Okita Y, Niwaya K (2003) Allograft replacement of common iliac artery mycotic aneurysm caused by Bacteroides fragilis vertebral spondylitis-a case report. Vasc Endovascular Surg 37: 441-444.

13. Santin M, Carratala J, Ariza X (1992) Hematogenous spondylitis caused by Bacteroides fragilis. Enferm Infecc Microbiol Clin 10: 380-381.

14. Doita M, Marui T, Kurosaka M (2001) Contained rupture of the aneurysm of common iliac artery associated with pyogenic vertebral spondylitis. Spine 26 e303-e307.

15. Elgouhari $H$, Othman M, Gerstein WH (2007) Bacteroides fragilis vertebral osteomyelitis: case report and a review of the literature. South Med J 100 506-511.

16. Salliot C, Lavie F, Azria A, Clerc D, Miquel A et al. (2005) Retroperitoneal fibrosis secondary to spondylodiscitis after infection with Prevotella. J Rheumatol 32 957-958.

17. Surbled M, Perrier-Creach C, Rabouille Y, Baladi G, Pedailles S (1992) Spondylodiscitis caused by Bacteroides melaninogenicus. Presse Med 21:1870-1871.

18. Anton E, Fernandez C, Barragan JM (2004) Spontaneous spondylodiscitis caused by bacteroides uniformis. Am J Med 117: 284-286.

19. Schober W, Horger M, Niehues D, Claussen CD, Duda SH (2003) One case of gram-negative anaerobic spondylodiscitis with Prevotella intermedia. Arch Orthop Trauma Surg 123: 436-438.

20. Crema MD, Pradel C, Marra MD, Arrive L, Tubiana JM (2007) Intramedullary spinal cord abscess complicating thoracic spondylodiscitis caused by Bacteroides fragilis. Skeletal Radiol Jul; 36: 681-683. 
21. Salavert M, Navarro V, Roig P, Gobernado M (1997) Vertebral osteomyelitis by Prevotella melaninogenica, Candida albicans and Mycobacterium tuberculosis in a intravenous drug addict. Enferm Infecc Microbiol Clin 15:117-119.

22. Lechiche C, Le MV, Marchandin H, Chanques G, Atoui N et al.( 2006) Spondylodiscitis due to Bacteroides fragilis: two cases and review. Scand Infect Dis 38: 229-231.

23. Saeed MU, Mariani P, Martin C (2005) Anaerobic spondylodiscitis: case series and systematic review. South Med J 98:144-148.

24. Li JS, Sexton DJ, Mick N (2000) Proposed modifications to the Duke criteria for the diagnosis of infective endocarditis. Clin Infect Dis 304: 633-638.

25. Thuny F, Grisoli D, Cautela J, Riberi A, Raoult D et al. (2014) Infective endocarditis: prevention, diagnosis, and management. Can J Cardiol 30: 10461057.

26. Saby L, Laas O, Habib G (2013) Positron emission tomography/computed tomography for diagnosis of prosthetic valve endocarditis: increased valvular 18F-fluorodeoxyglucose uptake as a novel major criterion. J Am Coll Cardiol 61: 2374-2382.

27. Raoult $D$ (2012) Chronic $Q$ fever: expert opinion versus literature analysis and consensus. J Infect Aug 65: 102-108. 